\title{
Application and Progress of Dexmedetomidine in Senile Anesthesia
}

\author{
Wu Yuqing, Hua Fuzhou, Lu Jun, Sun Jing and Chen Shoulin* \\ Department of Anesthesiology and Perioperative Medicine, the Second Affiliated Hospital of Nanchang University, Jiangxi \\ Nanchang 330006, China
}

*Corresponding author: Chen Shoulin, Department of Anesthesiology and Perioperative Medicine, the Second Affiliated Hospital of Nanchang University, Jiangxi Nanchang 330006, China

\begin{tabular}{|c|c|}
\hline ARTICLE INFO & ABSTRACT \\
\hline & $\begin{array}{l}\text { with the increase of the aging population, more and more elderly patients need } \\
\text { surgery. According to statistics, the number of operations was 310millions in } 2017 \text { in }\end{array}$ \\
\hline Published: 幽 February 11, 2021 & $\begin{array}{l}\text { the world, of which the proportion of operations for the elderly increased significantly to } \\
38.7 \% \text { [1]. The pathophysiological characteristics of the elderly are mainly characterized }\end{array}$ \\
\hline $\begin{array}{l}\text { Citation: Wu Yuqing, Hua Fuzhou, Lu Jun, } \\
\text { Sun Jing, Chen Shoulin. Application and } \\
\text { Progress of Dexmedetomidine In Senile } \\
\text { Anesthesia. Biomed J Sci \& Tech Res 33(5)- } \\
\text { 2021. BJSTR. MS.ID.005463. }\end{array}$ & $\begin{array}{l}\text { by the decrease of the function of the main organ system (nerve, respiration, } \\
\text { cardiovascular system, liver, kidney, etc.), and often coexist with a variety of chronic } \\
\text { diseases (hypertension, diabetes, coronary heart disease, etc.). Therefore, the selection } \\
\text { of anesthetic methods and anesthetic drugs for elderly patients should be cautious. } \\
\text { Dexmedetomidine hydrochloride (DEX) is a type of highly selective } \alpha 2 \text {-adrenergic } \\
\text { receptor agonist, which has good sedation, anti-anxiety, hypnosis, analgesia and anti- }\end{array}$ \\
\hline $\begin{array}{l}\text { Keywords: Dexmedetomidine; Senile } \\
\text { Anesthesia; Sedation and Analgesia; Organ } \\
\text { Protection; Cognitive Function }\end{array}$ & $\begin{array}{l}\text { sympathetic inhibition of perioperative stress response, and can stabilize hemodynamics } \\
\text { during anesthesia induction and maintenance. In recent years, many studies have } \\
\text { reported that Dexmedetomidine also has organ protective effects. The above advantages } \\
\text { of Dexmedetomidine are more suitable for the pathophysiological characteristics of } \\
\text { elderly patients, making it more widely used in anesthesia in elderly patients. Therefore, } \\
\text { the application and progress of Dexmedetomidine in senile anesthesia are reviewed in } \\
\text { this paper. }\end{array}$ \\
\hline
\end{tabular}

\section{Introduction}

\section{Pharmacological Characteristics}

Dexmedetomidine, a dextral isomer of metomidine, is a novel $\alpha$ 2-adrenergic receptor agonist with a receptor selectivity ( $\alpha$ 2: $\alpha 1$ ) of 1600 . Its affinity to $\alpha 2$-adrenergic receptor is 8 times higher than that of clonidine, and it is a highly selective and specific $\alpha$ 2-adrenoceptor agonist [1,2]. The central action of dexmedetomidine is mainly in the locus ceruleus of the brainstem, so it has the effect of sedation and inhibition of sympathetic activity. In addition, it also acts on the locus ceruleus and $\alpha 2$ receptors in the spinal cord to produce analgesic effect [3]. Dexmedetomidine mainly binds to $\alpha 2$ adrenergic receptors to exert physiological effects such as sedation, hypnosis, analgesia, sympathetic inhibition, neuroprotection, regulation of catecholamine release and so on. The most common route of administration of Dexmedetomidine is intravenous infusion, which has a high protein binding rate. $94 \%$ of Dexmedetomidine in plasma binds to albumin. In healthy volunteers, the distribution half-life of dexmedetomidine is about 6 minutes. The elimination half- life is 2.1 to 3.1 hours, mainly through liver metabolism [4,5]. The clearance rate of dexmedetomidine decreases with the severity of liver damage, so reducing the dose of Dexmedetomidine should be considered in patients with liver function damage. [6] The common adverse reactions of Dexmedetomidine were hypotension and bradycardia [7]. Some studies have reported hyperthermia during the administration of Dexmedetomidine in critically ill patients, which is highly indicative of drug fever. The mechanism of drug fever related to dexmedetomidine is not clear at present [8].

\section{Sedation and Analgesia}

Dexmedetomidine mainly acts on the central and peripheral nervous system, acts on $\alpha 2$ receptors in the brainstem-locus ceruleus, does not activate the GABA system, simulates natural non-eye movement sleep, and produces sedative effects. In addition, DEX can directly act on the spinal cord level of $\alpha 2$ receptors in the peripheral nerve and spinal dorsal horn, inhibit the transmission of pain signals to the brain, inhibit the transmission of nociceptive stimuli in the posterior horn of the spinal cord, and 
thus produce analgesic effect. The analgesic effect is independent of dose, and $1 \mathrm{ug} / \mathrm{kg}$ reaches the capping effect [9]. At present, most postoperative analgesics are opioids, which are easy to cause adverse reactions such as nausea, vomiting and pruritus in a dosedependent manner. The organ function of the elderly decreased and their tolerance to opioids was poor [10]. Dexmedetomidine can also be used as an adjuvant for postoperative analgesia. Many studies have shown that Dexmedetomidine has a good sedative and analgesic effect in elderly patients after operation. Lubin [11] and other studies have shown that the application of Dexmedetomidine before anesthesia can enhance the effect of sedation and analgesia in elderly patients undergoing abdominal tumor surgery, reduce the incidence of adverse reactions such as respiratory depression, nausea and vomiting, and improve the comfort of elderly patients. There are studies to compare the sedative and analgesic effects of ketamine-Dexmedetomidine and ketamine-propofol in patients after coronary artery bypass grafting (CABG). Results the extubation time of ketamine-Dexmedetomidine group was shorter, and the analgesic effect of ketamine-Dexmedetomidine group was better than that of ketamine-propofol group [12].

Dexmedetomidine can not only exert the effect of sedation and analgesia through intravenous administration, but also be used for epidural administration. A systematic review and meta-analysis of dexmedetomidine as an epidural analgesia and anesthetic adjuvant found that epidural injection of Dexmedetomidine is safe and effective, and can prolong the time of analgesia, and the sedation score is significantly increased [13].

\section{Organ Protection}

In the research reports on dexmedetomidine, in addition to affirming its sedative and analgesic effects, more and more studies have found that dexmedetomidine may protect a variety of organs through mechanisms such as inflammatory response, oxidative stress, apoptosis and so on.

\section{Spinal Cord Protection}

Spinal cord ischemia-reperfusion injury mainly occurs after the operation of spine and thoracic and abdominal arteries, which can cause unpredictable complications and even paraplegia [14]. Spinal cord ischemia-reperfusion injury is a pathophysiological process in which multicellular and multi-mediators play a role together, and its main mechanisms are oxidative stress, inflammatory response and apoptosis. In the study of Fang B [15], it was confirmed that dexmedetomidine could reduce the damage of blood spinal cord barrier induced by spinal cord ischemia-reperfusion injury in aged rats, and its mechanism was mediated by inhibition of MMP-9 and enhancement of Ang1-Tie2 system. Excessiveinflammatory response plays an important role in spinal cord ischemia-reperfusion injury, and the anti-inflammatory effect of Dexmedetomidine can inhibit excessive inflammatory response. HuiRong et al. [16] studied the effects of dexmedetomidine preconditioning on pro-inflammatory and anti-inflammatory systems after spinal cord injury in rats. It was found that:

a) Spinal cord ischemia-reperfusion injury led to a significant increase in the expression of TLR 4 and $\alpha 7 \mathrm{nAChR}$.

b) dexmedetomidine inhibited the expression of TLR4, but significantly increased the expression of $\alpha$ nAChR.

c) the downregulation of TLR4 induced by dexmedetomidine needs to activate 7nAChR/PI3K/Akt/PU.1 pathway at least to some extent.

d) theupregulation of $\alpha 7 \mathrm{nAChRinduced} \mathrm{by} \mathrm{dexmedetomidine}$ requires the activation of a2R/PI3K/Akt/Ly6h pathway. These mechanisms may be the basis that dexmedetomidine can reduce apoptosis and promote the recovery of motor function in rats with spinal cord injury. LiuJ [17] and other researchers have found that Dex preconditioning can protect the spinal cord from ischemia / reperfusion injury by inhibiting HMGB1TLR4-NF-KB signal pathway, thereby inhibiting inflammatory response and stabilizing the integrity of the blood-spinal cord barrier. Dexmedetomidine also increases the number of astrocytes in the spinal cord by activating $\alpha 2$ receptors, and astrocytes protect the spinal cord by releasing neurotrophic factors to maintain the activity of neurons $[18,19]$ and other experiments found that Dexmedetomidine can reduce spinal cord ischemia-reperfusion injury by blocking mast cell degranulation. Dexmedetomidine can protect spinal cord ischemia-reperfusion injury through a variety of mechanisms and can play a better spinal cord protection role in thoracic and abdominal arteries and spinal surgery in elderly patients, so as to reduce a series of postoperative complications.

\section{Lung Protection}

With the development of laparoscopic technology and the rise of the concept of rapid rehabilitation, laparoscopy is often the first choice for abdominal surgery. However, during laparoscopic surgery, $\mathrm{CO}_{2}$ pneumoperitoneum can increase intraabdominal pressure and move the diaphragm upward, resulting in obstruction of chest and lung activity. High tidal volume of traditional mechanical ventilation leads to a significant increase in intrathoracic pressure and a sharp increase in airway pressure [20], resulting in a decrease in chest and lung compliance, pulmonary collapse and expansion, alveolar ventilation, decreased functional residual capacity, and atelectasis. In severe cases, it may lead to imbalance of pulmonary ventilation blood flow ratio and injury of lung tissue [21]. Obvious changes in pulmonary compliance and airway pressure during operation can lead to damage of oxygenation function and ventilatory function. The increase of intrathoracic pressure will lead to lung ischemia, when the pneumoperitoneum is released, the ischemic lung tissue is reperfused, and the oxygen free radicals produced by ischemic lung tissue will cause secondary injury in the process of reperfusion. Dexmedetomidine not only produces anesthetic effect, but also plays a protective role in lung injury by inhibiting inflammatory factors and related proteins through antioxidation. In order to 
explore the protective effect of dexmedetomidine on lung tissue during laparoscopic surgery, Geze S [22] and others have done related experiments. The results suggest that dexmedetomidine preconditioning has a protective effect on lung tissue injury caused by pneumoperitoneum. Cardiopulmonary bypass ((CPB)) plays an important role in the treatment of patients with cardiac surgery, macrovascular surgery and cardiopulmonary failure [23-24], but as a non-physiological mode of circulation, the injury of lung function caused by CPB is more common [25-26]. Some studies have found that dexmedetomidine can effectively reduce lung injury in rats during cardiopulmonary bypass. Its mechanism is to activate P13K/Akt signal pathway, inhibit downstream Caspase protein expression, and reduce lung injury [27-29].

\section{Myocardial Protection}

When myocardial perfusion is restored after a certain period of ischemia, the damage in metabolism, function and structure will be further aggravated. the severity of the occurrence is related to tissue oxygen demand, tolerance to ischemia and hypoxia, ischemia duration, collateral circulation and other factors [30]. Most elderly patients are complicated with hypertension, diabetes, coronary heart disease and other basic diseases, these diseases themselves increase the burden of the heart, damage the myocardium to a certain extent, and can maintain the relative stability of hemodynamics at rest, but general anesthesia, endotracheal intubation and extubation, surgical stimulation will lead to hemodynamic fluctuations and myocardial injury [31]. YuJ and other studies found that Dex preconditioning has a protective effect on cardiac ischemiareperfusion injury by down-regulating the expression of miR-665 and up-regulating the expression of AK1 and Cnr2 [32]. Youting $\mathrm{Ju}$ [33] found that Dexmedetomidine protects myocardium from ischemia-reperfusion injury through cholinergic anti-inflammatory pathways. DongJing [34] explored the intraperitoneal injection of dexmedetomidine before ischemia-reperfusion in aged rats, and found that Dexmedetomidine preconditioning can effectively reduce the area of cardiac ischemia-reperfusion injury in aged rats. Shoulin Chen et al. studied 64 patients undergoing elective coronary artery bypass graft surgery under cardiopulmonary bypass. The results showed that continuous intravenous infusion of dexmedetomidine during anesthesia could alleviate myocardial injury and attenuate the systemic inflammatory response in patients under cardiopulmonary bypass [35].

\section{Intestinal Protection}

Pathophysiological conditions such as intestinal obstruction, trauma, shock and surgical stress can lead to intestinal ischemiareperfusion injury [36]. The elderly have a high incidence of intestinal obstruction. Intestinal obstruction itself can cause intestinal mucosal ischemia and necrosis, which leads to the damage of intestinal barrier function. bacteria and toxins in the intestine can reach mesenteric lymphoid tissue, lymph, blood and extra-intestinal organs through the damaged intestinal mucosa. It leads to intestinal sepsis and finally leads to multiple organ dysfunction syndrome [37]. Therefore, the prevention of intestinal ischemia-reperfusion injury is particularly important to improve the prognosis of patients and reduce mortality [38]. Reported that intraperitoneal injection of dexmedetomidine can inhibit inflammatory reaction, inhibit intestinal epithelial cell apoptosis, maintain the integrity of intestinal structure, and has obvious protective effect on intestinal ischemia-reperfusion injury in rats [39]. Studied the effect of Dexmedetomidine at different time and dose on intestinal ischemia-reperfusion injury in rats. The results showed that Dexmedetomidine attenuated intestinal ischemia-reperfusion injury by activating adrenergic a2 receptor and inhibiting inflammatory reaction and intestinal epithelial cell apoptosis. Believe that dexmedetomidine protects the intestinal tract from ischemia-reperfusion injury, at least to some extent, increases the activity of antioxidant enzymes and cell proliferation, and inhibits the protein levels of caspase-3, MCP, PAR2 and the activation of JAK/STAT signal pathway induced by injury [40] the down-regulation of telomerase and caspase-3mRNA may be involved in the protective effect of DEX on intestinal ischemiareperfusion injury [41].

\section{Renal Protection}

Ischemia-reperfusion injury is a common postoperative complication in perioperative patients, which can lead to death in severe cases. Patients who undergo long-term major surgery, abdominal surgery and genitourinary surgery and suffer from risk factors such as advanced age, peripheral artery disease, diabetes, renovascular disease and congestive heart failure are more likely to cause acute renal injury due to impaired renal perfusion and decreased function [42]. Therefore, how to avoid perioperative renal ischemia-reperfusion injury has become the focus of scholars at home and abroad. In recent years, a large number of studies have shown that Dexmedetomidine has a protective effect on renal ischemia-reperfusion injury [43]. Some studies have shown that dexmedetomidine preconditioning may reduce cell death and HMGB1 release through a2 receptor activation of PI3K/pAKT cell signal pathway, and inhibit TLR4 signal, thus reducing renal ischemia-reperfusion injury $[44,45]$. Made a histopathological study on the effect of Dexmedetomidine on renal ischemia-reperfusion injury in rats. They found that Dexmedetomidine significantly reduced the degree of edema in glomeruli and tubules by reducing renal histological changes caused by ischemia-reperfusion injury [46]. Detected the apoptosis of renal tissue in the rat model of ischemia-reperfusion injury, and found that the histopathological structure of rats treated with Dexmedetomidine was better and the degree of apoptosis was lower. Sayana [47], using contrastenhanced ultrasound quantitative analysis technique combined with pathological examination, found that Dexmedetomidine could improve microcirculation perfusion during renal ischemia / reperfusion injury in rabbits. The protective effect of dexmedetomidine on kidney may be through anti-inflammatory 
reaction, antioxidant stress, anti-apoptosis, inhibition of renal microvasoconstriction and other mechanisms [48].

\section{Perioperative Neurocognitive Impairment (PND)}

Postoperative cognitive dysfunction is the most common complication of central nervous system after general anesthesia, especially in elderly patients. PND will affect the prognosis of surgical patients, and even affect the long-term survival of patients. With the advent of the aging population, PND is an urgent problem to be solved. At present, many studies have shown that dexmedetomidine has a certain effect on the prevention of postoperative delirium in elderly patients, such as [49]. A doubleblind randomized controlled study on the effect of time and dose factors of dexmedetomidine on postoperative delirium in elderly patients after laparoscopic major surgery found that intraoperative continuous injection of Dexmedetomidine can reduce the incidence of postoperative delirium more than postoperative continuous injection of dexmedetomidine. A meta-analysis of the effect of dexmedetomidine on postoperative cognitive impairment [50] concluded that perioperative treatment of dexmedetomidine could significantly reduce the incidence of POCD and inflammation and improve postoperative neurocognitive function compared with normal saline control group and anesthesia control group [51] selected elderly patients who underwent carotid endarterectomy as the research object and selected inflammatory mediators and neurotrophic factor (BDNF) as the research index.

The experimental results showed that Dexmedetomidine could improve the cognitive impairment of elderly patients after carotid endarterectomy by inhibiting cerebral inflammation and enhancing the expression of brain-derived neurotrophic factor. The effect of dexmedetomidine on improving postoperative cognitive function in elderly patients is affirmative. Some scholars have conducted a series of studies on the mechanism of improving postoperative cognitive function, such as [51] animal experiments found that dexmedetomidine can reduce postoperative cognitive dysfunction in elderly rats may play a role through SIRT1 signal pathway [52] to explore the mechanism of dexmedetomidine in improving postoperative cognitive dysfunction from the point of view of neuronal mitochondrial membrane oxidative stress and electrophysiological dysfunction. Studies have found that Dexmedetomidine can reduce neuronal damage, which may be due to reducing mitochondrial membrane oxidative stress, reducing the damage of mitochondrial related enzyme activity, and reducing the damage of mitochondrial respiratory chain enzyme complex, and finally improving the patients with postoperative cognitive impairment [52] found that the protective effect of Dex on POCD may be through inhibiting GABABR, up-regulating cAMP-PKA-CREB signal pathway, thus reducing hippocampal inflammation caused by surgical trauma.

\section{Summary}

Most studies have confirmed that dexmedetomidine has the effects of analgesia and sedation, inhibition of sympathetic nerve and organ protection, and has a good effect in clinical anesthesia. While taking into account the sedation, analgesia and organ protection of dexmedetomidine, we can explore different administration times, appropriate doses and strategies of Dexmedetomidine, so as to provide more reliable guidance for clinical use in the future. In addition, the effect of DEX combined with other drugs is also worthy of in-depth study.

\section{Acknowledgement}

This review was supported by Subject of Jiangxi Provincial Education Department (GJJ200180).

\section{References}

1. McDade E, Bateman RJ (2017) Stop Alzheimer's before it starts. Nature 547: 153-155

2. Xu Fei, ao Hushan (2015) Clinical application of Dexmedetomidine hydrochloride. Chinese Journal of Circulation 30(04): 401-403.

3. Xie Shouyu, Ma Ke (2017) Progress in drug treatment of perioperative acute pain. Chinese Journal of pain Medicine 23(08): 608-612.

4. Weerink MAS, Struys MMRF, Hannivoort LN, Barends CRM, Absalom AR, et al. (2017) Clinical Pharmacokinetics and Pharmacodynamics of Dexmedetomidine. Clin Pharmacokinet 56(8): 893-913.

5. Lee S, Kim BH,Lim K,D Stalker, W Wisemandle, et al. (2012) Pharmacokinetics and pharmacody namics of intravenous dexmedetomidine in healthy Korean sub ects. J Clin Pharm Ther 37(6): 698-703.

6. Xu Rongrong, Huang Wenqi (2012) Advances in pharmacokinetics and pharmacodynamics of Dexmedetomidine. Guangdong medicine 33(08): 1037-1040.

7. Wang Fu, Chen Yinglu, Zhang Liang (2019) Research progress on the effects of different administration routes of Dexmedetomidine on the synergistic effect of regional anaesthesia. Chinese pharmacy 30(16): 2297-2304.

8. Krüger BD, Kurmann J, Corti N, Spahn DR, Bettex D, et al. (2017) Dexmedetomidine-Associated Hyperthermia: A Series of 9 Cases and a Review of the Literature. Anesth Analg 125(6): 1898-1906.

9. Xie Shouyu, Ma Ke (2017) Progress in drug treatment of perioperative acute pain. Chinese Journal of pain Medicine 23(08): 608-612.

10. Wang Xiaoting, Zheng Lihong (2015) Research progress on the application of Dexmedetomidine in anesthesia in the elderly. Journal of practical Medicine 31(23): 3965-3967.

11. Lu Bin, Zheng Ye Ying, Zhang Juntao (2017) Effect of dexmedetomidine before anesthesia induction on postoperative analgesia and sedation in elderly patients with abdominal tumor. Chinese Journal of Gerontology 37(16): 4033-4035.

12. Mogahd MM, Mahran MS, Elbaradi GF (2017) Safety and efficacy of ketamine-dexmedetomidine versus ketamine-propofol combinations for sedation in patients after coronary artery bypass graft surgery. Ann Card Anaesth. 20(2): 182-187.

13. Zhang X, Wang D, Shi M, Luo Y (2017) Efficacy and Safety of Dexmedetomidine as an Adjuvant in Epidural Analgesia and Anesthesia: A Systematic Review and Meta-analysis of Randomized Controlled Trials. Clin Drug Investig 37(4): 343-354. 
14. Awad H, Ramadan ME, El Sayed HF, Tolpin DA, Tili E, et al. (2017) Spinal cord injury after thoracic endovascular aortic aneurysm repair. Can J Anaesth 64(12): 1218-1235.

15. Fang B, LiXQ, BiB, TanWF, LiuG, etal.(2015) Dexmedetomidine attenuates blood-spinal cord barrier disruption induced by spinal cord ischemia reperfusion injury in rats. Cell Physiol Biochem 36(1): 373383.

16. Rong H , Zhao Z , Feng J , Yishan Lei, Hao Wu, et al. (2017) The effects of dexmedetomidine pretreatment on the pro- and anti-inflammation systems after spinal cord injury in rats. Brain, Behavior, and Immunity 64: 195-207.

17. LiuJ, ZhangS, FanX, YuanF, Dai J, etal.(2019) Dexmedetomidine Preconditioning Ameliorates Inflammation and Blood-Spinal Cord Barrier Damage After Spinal Cord Ischemia-Reperfusion Injury by DownRegulation High Mobility Group Box 1-Toll-Like Receptor 4-Nuclear Factor $\kappa B$ Signaling Pathway. Spine (Phila Pa1976) 44(2): E74-E81.

18. Freeman KA, Fullerton DA, Foley LS, Bell MT, Cleveland JC Jr, et al. (2015) Spinal cord protection via alpha-2agonist mediated increase in glial cell-line-derived neurotrophic factor. J Thorac Cardiovasc Surg 149(2): 578-584

19. Ma J, Zhang XL, Wang CY, Lin Z, Tao JR, et al. (2015) Dexmedetomidine alleviates the spinal cord ischemia-reperfusion injury through blocking mast cell degranulation. Int J Clin Exp Med 8(9): 14741-14749.

20. Chia-Chih Liao, Yi-Chuan Kau, Pei-Chi Ting, Shih-Chang Tsai, ChinJung Wang, et al. (2016) The Effects of Volume-Controlled and PressureControlled Ventilation on Lung Mechanics, Oxidative Stress, and Recovery in Gynecologic Laparoscopic Surgery. The Journal of Minimally Invasive Gynecology 23(3): 410-417.

21. Wirth S, Biesemann A, Spaeth J, Stefan Schumann (2017) Pneumoperitoneum deteriorates intratidal respiratory system mechanics: an observational study in lung-healthy patients. Surgical Endoscopy 31(2): 753-760.

22. Geze S, Cekic B, Imamoğlu $M$, Yörük $M F$, Yuluğ $E$, et al. (2012) Use of dexmedetomidine to prevent pulmonary injury afterpneumoperitoneum in ventilated rats. Surg Laparosc Endosc Percutan Tech 22(5): 447-453.

23. Liu Xi, Chen Xun, Zhao Zhou (2014) Analysis of prognosis, causes and risk factors of external circulation during off-pump coronary artery bypass grafting. Chinese Journal of Circulation 29(11): 879-883.

24. Shi H, Lu RJ, Wang S, Honglin Chen, Fei Wang et al. (2017) Effects of SDF-1/CXCR4 on acute lung injury induced by cardiopulmonary bypass . Inflammation 40(3): 937-945.

25. Feng JB, Liu HY, Chen JY, Jiyuan Wang, Zhuang Liu et al. (2016) Levosimendan reduces lung injury in a canine model of cardiopulmonary bypass . Korean Circ J 46(3): 402-407.

26. Meng F, Han J, Wang J, Haibo Zhang, Chunlei Xu et al. (2018) The gender-specific expression of neuropeptide $Y$ and neuropeptide $\mathrm{Y}$ receptors in human atrial tissue Dduring cardiopulmonary bypass surgery . J Thorac Dis10(12): 6563-6568.

27. Cao Jie, Zhang Eryong (2019) Signal pathway related to the protective effect of dexmedetomidine on lung tissue after ischemia-reperfusion injury during cardiopulmonary bypass. Journal of Southern Medical University 2019(08): 980-986.

28. Cao G, Zhang E (2019) [Protective effects of dexmedetomidine against pulmonary ischemia-reperfusion injury during cardiopulmonary bypass in rats]. Nan Fang Yi Ke Da Xue Xue Bao 39(8): 980-986.

29. Li J, Dou X, Li D, He M, Han M, et al. (2019) Dexmedetomidine Ameliorates Post-CPB Lung Injury in Rats by Activating the PI3K/Akt Pathway. J Invest Surg 26: 1-8.

30. Zhang Jie, Xiong Wei, Pu Yuju, Qian Jinqiao (2019) Research progress on the mechanism of myocardial protection of dexmedetomidine. Medical review 25(14): 2863-2867.
31. Li Zhiqiang (2010) Effect of Dexmedetomidine on perioperative hemodynamics in elderly patients with hypertension under general anesthesia. Journal of Hubei University for nationalities (Medical Edition) 27(03): 22-24.

32. Yu J, Yang W, Wang W, Wang Z, Pu Y, et al. (2019) Involvement of miR-665 in protection effect of dexmedetomidine against Oxidative Stress Injury in myocardial cells via CB2 and CK1. Biomed Pharmacother 115: 108894.

33. Youting Ju, Fan Xiao, Jun Lu, Bin Zhou 2, Junying Cai, et al. (2020) Effect of dexmedetomidine and cholinergic anti-inflammatorypathways in myocardial ischemia-reperfusion injury . Pak J Pharm Sci 33(3(Special)): 1377-1382.

34. Dong J , Guo X, Yang S, Lin Li (2017) The effects of dexmedetomidine preconditioning on aged rat heart of ischaemia reperfusion injury. Research in Veterinary Science 114: 489-492.

35. Chen SL, Jun Lu, Yu Jiang, Yan Hua Tang, Lei Tao, et al. (2015) Effect of dexmedetomidine on myocardial ischemia-reperfusion injury. Int J Clin Exp Med 8(11): 21166-21172.

36. Yao Biyu, Chen Yanglei, Li Songting (2015) Rosiglitazone preconditioning attenuated intestinal ischemia-reperfusion injury [J. Modern Applied Pharmacy in China 32(6): 676-681.

37. Mantz J, Josserand J, Hamada S (2011) Dexmedetomidine: new insights. Eur J Anaesthesiol 28(1): 3-6.

38. Zhang X, Zhou X, Zhang Q, Feng Zhu (2015) The preventive effects of dexmedetomidine against intestinal ischemia-reperfusion injury in Wistar rats. Iranian Journal of Basic Medical Science 18(6): 604-609.

39. Zhang X Y, Liu Z M, Wen S H, Yun-Sheng Li, Yi Li, et al. (2012) Dexmedetomidine Administration before, but Not after, Ischemia Attenuates Intestinal Injury Induced by Intestinal Ischemia-Reperfusion in Rats. Anesthesiology 116(5): 1035-1046.

40. Zhang XK, Zhou XP, Zhang Q, Zhu F (2015) The preventive effects of dexmedetomidine against intestinal ischemia-reperfusion injury in Wistar rats. Iran J Basic Med Sci 18(6): 604-609.

41. Sun Y, Gao Q, Wu N, Li SD, Yao JX, et al. (2015) Protective effects of dexmedetomidine on intestinal ischemia-reperfusion injury. Exp Ther Med 10(2): 647-652.

42. Erkılıç, Ezgi, Kesimci E , Alaybeyoglu F, Tural R, et al. (2017) Does remifentanil attenuate renal ischemia-reperfusion injury better than dexmedetomidine in rat kidney?. Drug Design Development and Therapy 11: 677-683.

43. Liu Y, Sheng B , Wang S , Feiping Lu, Jie Zhen, et al. (2018) Dexmedetomidine prevents acute kidney injury after adult cardiac surgery: a meta-analysis of randomized controlled trials. BMC Anesthesiology 18(1): 7.

44. Gu J, Sun P, Zhao H, Helena R Watts, Robert D Sanders, et al. (2011) Dexmedetomidine provides renoprotection against ischemiareperfusion injury in mice. Critical Care 15(3): R153.

45. Kocoglu H, Ozturk H, Ozturk H, Fahri Yilmaz, Nebahat Gulcu, et al. (2009) Effect of dexmedetomidine on ischemia-reperfusion injury in rat kidney: a histopathologic study. Renal Failure 31(1): 70-74.

46. Si Yanna, Hallyu, Zhang Yuan, Chen Lihai, Xu Yajie, et al. (2016) Contrastenhanced ultrasonography to evaluate the effect of dexmedetomidine on microcirculation perfusion during renal ischemia / reperfusion injury in rabbits . Journal of Southern Medical University 36(05): 628-632.

47. Zhao Tianshou (2018) Research progress on the renal protective effect of Dexmedetomidine and its mechanism. Popular science and technology 2018.

48. Lee C , Lee C H , Lee G, Myeongjong Lee, Jihyo Hwang et al. (2018) The effect of the timing and dose of dexmedetomidine on postoperative delirium in elderly patients after laparoscopic major non-cardiac surgery: A double blind randomized controlled study. Journal of Clinical Anesthesia 47: 27-32 
49. Li WX, Luo RY, Chen C, Li X, Ao JS, et al. (2019) Effects of propofol, dexmedetomidine, and midazolam on postoperative cognitive dysfunction in elderly patients: a randomized controlled preliminary trial. Chin Med J (Engl) 132(4): 437-445.

50. Fang S, Chen Y, Yao P, Li Y, Yang Y, et al. (2018) Dexmedetomidine alleviates postoperative cognitive dysfunction in aged rats probably via silent information regulator 1 pathway. Nan Fang Yi Ke Da Xue Xue Bao 38(9): 1071-1075.

51. Chen J, Shen N, Duan X, Guo Y (2018) An investigation of the mechanism of dexmedetomidine in improving postoperative cognitive

\section{ISSN: 2574-1241}

DOI: 10.26717/BJSTR.2021.33.005463

Chen Shoulin. Biomed J Sci \& Tech Res

(C) This work is licensed under Creative

Submission Link: https://biomedres.us/submit-manuscript.php dysfunction from the perspectives of alleviating neuronal mitochondrial membrane oxidative stress and electrophysiological dysfunction. Exp Ther Med 15(2): 2037-2043.

52. Zhu YS, Xiong YF, Luo FQ, Min J (2019) Dexmedetomidine protects rats from postoperative cognitive dysfunction via regulating the GABA(B) R-mediated cAMP-PKA-CREB signaling pathway. Neuropathology 39(1): 30-38.

$\begin{array}{ll}\text { BIOMEDICAL } & \text { Assets of Publishing with us } \\ \text { RESEARCHES } & \text { - Global archiving of articles } \\ & \text { - Immediate, unrestricted online access } \\ & \text { - Rigorous Peer Review Process } \\ & \end{array}$

